\title{
Finite element-based model for crack propagation in linear elastic materials
}

\author{
Abdulnaser M. Alshoaibi ${ }^{a^{*}}$
}

${ }^{a}$ Department of Mechanical Engineering, Jazan University, P. O. Box 706, Jazan 45142, Kingdom of Saudi Arabia

\begin{tabular}{l}
\hline A R T I C L EI N F O \\
\hline Article history: \\
Received 12 August 2019 \\
Accepted 14 October 2019 \\
Available online \\
14 October 2019 \\
\hline Keywords: \\
Crack growth \\
Finite element \\
Stress intensity factors \\
Linear elastic fracture \\
mechanics \\
Adaptive Mesh generation
\end{tabular}

\section{Introduction}

Have a think what would occurs in a test specimen when a crack is loaded, if starting by applying a small load to the crack then it will begin to open and a small plastic zone forms at the tip of the crack, increasing the load increases the size of the plastic zone. Eventually the load will reach to critical value and the crack will extend forward into the plastic zone then the material will be deformed due to crack growth also that leads to fracture. Although fracture is a common and ubiquitous occurrence, it is a failure mode that remains difficult to predict. Fracture behavior is heavily dependent on loading conditions, component geometry, and material properties. Often one will find that a material that is resistant to cracking under one set of conditions can fracture easily under another. Additionally, cracking can occur without warning and come with catastrophic consequences. Experimental validation of component fracture resistance is not only expensive, but time consuming and often impractical if the loading conditions are difficult to recreate in a laboratory setting. The ability to accurately model and predict cracking behavior is therefore valuable and necessary in light of the growing complexity and performance demands of modern applications. The capability to predict brittle fracture of engineering structures in manufacturing and service are very important. Stress intensity factors are important for fracture analysis

* Corresponding author.

E-mail addresses: alshoaibi@gmail.com (A. M. Alshoaibi)

(C) 2020 Growing Science Ltd. All rights reserved.

doi: $10.5267 /$ j.esm.2019.10.002 
of cracked structures, because it have been used for the prediction of crack initiation, crack growth direction and fracture speed in brittle materials. In recent decades, various methods including experimental test, numerical analysis and simulation have been developed, from which finite element method (FEM) calculation can provide more convenient means for optimal design. Zhang and Guo (2018) proposed a singular Voronoi cell finite element model for estimation of the mixed mode stress intensity factors (SIFs) of crack tip based on a modified complementary energy principle. In the finite element simulation the discretization of a domain was initially defined, based on the meshes. The mesh size sensitivity of this method in analyzing problems especially problems related to moving boundaries, such as crack propagation (Guo, Xiang et al. 2016), and the need to define adaptive fine mesh around the crack tip inspired researchers to present a mesh-less or mesh-free method. A mesh-less method uses a number of scattered nodes within the domain and on the boundaries. The most essential mesh-less methods for analyzing problems with moving boundaries like crack propagation are the Element-Free Galerkin Method (EFGM) (Belytschko et al. 1994) and $h-p$ clouds (Duarte and Oden 1996) . It is importance saying that these methods entail high computational costs. Therefore, under similar conditions, mesh-less methods are much more expensive than FEM. For this reason, in Rao and Rahman (2001), a combination of an FEM and a mesh-less method has been utilized to alleviate computational costs without a loss of accuracy.

Sabbagh-Yazdi et al. (2018) proposed a new approach to model two-dimensional linear crack propagation, based on the Galerkin Finite Volume Method. Azocar et al. (2010) represented that finite element based numerical method applied to fracture mechanics proceeds iteratively as follows: an approximate displacement field solution is numerically obtained; then a numerical approximation of the fracture parameters is computed by using appropriate data post processing. In any case, mesh generation is a critical aspect of an efficient crack propagation method. This should consider at least the following issues: firstly, generation of a good quality initial mesh of the complex geometry such as a cracked one. A crack in a 2D geometry is represented as a 1D entity, where two free surfaces coincides geometrically but are topologically different, thus the mesh generator must be able to take into account these free surfaces, both in the initial mesh generation and in the crack growth steps. Secondly, in a cracked geometry mesh, elements near the crack tip are much smaller than elements far from crack tip, so the algorithm must generate a good size transition between these zones, optimizing element size and keeping element quality in all the mesh. Third, crack propagation simulation implies modification of both the object geometry and its associated mesh for every time step. This paper presents the application of FEM for the analysis of crack growth problems and the correlation of results with experimental data.

\section{Crack Growth Direction Criteria and Mesh Refinements}

Typical progressive fracture simulation algorithms update the modeled geometry during each simulation step with a small straight-line crack advance segment. In a linear-elastic isotropic two dimensional model, the direction of each straight-line propagation segment can be calculated by assuming a propagation direction criterion and determining two parameters that characterize a first-order near-tip Williams stress field. Conventionally, these two parameters are the first-order symmetric mode I, and anti-symmetric mode II, stress intensity factors $\left(K_{\mathrm{I}}, K_{\mathrm{II}}\right)$, computed for a crack advancing in its current self-similar (r) direction (Williams 1961). Adaptive subdomain re-meshing techniques add each crack advance segment by modifying the current finite element mesh. This is done by removing elements and nodes in a region ahead of the current crack tip and replacing them with smaller elements along the crack faces to be added. These additional elements typically include a small structured rosette of elements at the new crack tip location. The remaining space between is filled with additional elements. Insufficiently robust mesh modification algorithms can cause premature termination of a progressive fracture simulation (Miller et al. 2015). Only a geometric description of a crack path needs to be updated during each simulation step because cracks are not represented explicitly in an adaptive mesh. This direct method has been extended to represent multiple cracks without the need for mesh refinement between crack tips (Lan et al. 2013). 
The maximum circumferential stress or strain criterion, the maximum energy release rate criterion, the minimum strain energy density criterion and the maximum circumferential stress criterion can be used to determine the direction of crack propagation, as stated in different papers (Belytschko \& Black, 1999; Ayatollahi et al., 2006, 2011; Khan \& Khraisheh 2000, 2004; Ayatollahi \& Aliha 2007, 2009; Mirsayar et al., 2014, 2018, Akbardoost et al., 2014; Aliha et al., 2010, 2016a,b, 2017; Razavi et al., 2018, AbdElhady, 2013; Aliha \& Ayatollahi 2008, 2012, Xie \& Waas 2006; Aliha \& Saghafi 2013; Dehghany et al., 2017, Sutton et al., 2000; Cooke \& Pollard, 1996; Aliha \& Rezaei, 2011; Matvienko, 2012). In present study the maximum circumferential stress criterion is exploited for isotropic materials under mixed-mode loading.

The crack propagation is observed in normal direction of maximum tangential tensile stress given by

$$
\sigma_{\theta}=\frac{1}{\sqrt{2 \pi r}} \cos \frac{\theta}{2}\left[K_{I} \cos ^{2} \frac{\theta}{2}-\frac{3}{2} K_{I I} \sin \theta\right]
$$

The normal direction to the maximum tangential stress can be obtained by solving $d \sigma_{\theta} / d \theta=0$ for $\theta$. The nontrivial solution is expressed as

$$
K_{I} \sin \theta+K_{I I}(3 \cos \theta-1)=0
$$

where $\theta$ is given by

$$
\theta= \pm \cos ^{-1}\left\{\frac{3 K_{I I}^{2}+K_{I} \sqrt{K_{I}^{2}+8 K_{I I}^{2}}}{K_{I}^{2}+9 K_{I I}^{2}}\right\}
$$

\section{Stress Intensity Factors Method}

In Rice (1968) introduced $J$-integral to study non-linear material behavior in small scale yielding. It is a path independent contour integral defined as:

$$
J=\int_{c}\left[W n_{1}-\sigma_{i j} n_{j} \frac{\partial u_{i}}{\partial x}\right] d s,
$$

where strain-energy density is denoted by $\mathrm{W}$; stresses by $\sigma_{i j}$; the displacements corresponding to local $i$-axis $u_{i}$; arc length of the contour is expressed as $s ; n_{j}$ is the unit outward normal to the contour $\mathrm{C}$, surrounding the crack tip (Fig. 1a).

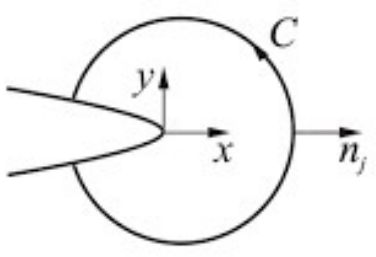

(a)

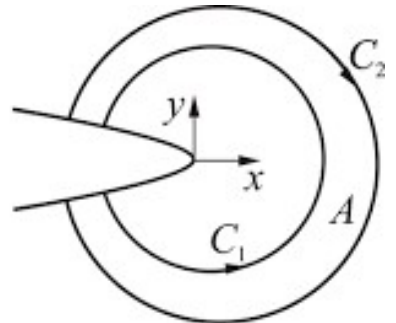

(b)

Fig. 1. (a) Arbitrary contour containing the crack tip; (b) Area to calculate the $J$-integral. 
The Equivalent Domain Integral Method being more convenient for finite element analyses replaces finite size domain by the divergence theorem by the integration along the contour. For two-dimensional problems area integral replaces the contour integral i (Fig. 2b). Then, Eq. (4) is rewritten as

$$
J_{k}=-\int_{A}\left[W \frac{\partial q}{\partial x}-\sigma_{i j} \frac{\partial u_{i}}{\partial x} \frac{\partial q}{\partial x}\right] d A-\int_{A}\left[\frac{\partial W}{\partial x}-\frac{\partial}{\partial x}\left(\sigma_{i j} \frac{\partial u_{i}}{\partial x}\right)\right] q d A-\int_{s} t_{i} \frac{\partial u_{i}}{\partial x} q d s
$$

In linear elastic analysis, the $J$-integral by definition take account of translational mechanical energy balance in front of the crack along the $x$-axis. In either cases of pure Mode I and Mode II, Eq. (5) facilitate the calculation of the stress intensity factors $K_{I}$ or $K_{I I}$ but fails for mixed mode condition to calculate $K_{I}$ and $K_{I I}$ separately. For such case invariant integrals are used as defined by Knowles and Sternberg (1972).

$$
J_{k}=-\int_{A}\left[W \frac{\partial q}{\partial x_{k}}-\sigma_{i j} \frac{\partial u_{i}}{\partial x_{k}} \frac{\partial q}{\partial x_{j}}\right] d A-\int_{A}\left[W \frac{\partial q}{\partial x_{k}}-\sigma_{i j} \frac{\partial}{\partial x_{j}}\left(\frac{\partial u_{i}}{\partial x_{k}}\right)\right] q d A-\int_{s} t_{i} \frac{\partial u_{i}}{\partial x_{k}} q d s,
$$

where $k$ is an index for local crack tip axis $(x, y)$. These integrals were introduced initially for small deformation and were extended by Atluri (1982) for finite deformation.

The stress intensity factors can be obtained by two possible ways. The first approach is through relationships between the $J$-integral and the stress intensity factors. These relations are:

$$
J_{1}=\frac{\kappa+1}{8 \mu}\left(K_{I}^{2}+K_{I I}^{2}\right), J_{2}=-\frac{\kappa+1}{4 \mu} K_{I} K_{I I} .
$$

Then the relations between the stress intensity factors and $J_{1}, J_{2}$ are:

$$
K_{I}=0.5 \sqrt{\frac{8 \mu}{\kappa+1}}\left(\sqrt{J_{1}-J_{2}}+\sqrt{J_{1}+J_{2}}\right), K_{I I}=0.5 \sqrt{\frac{8 \mu}{\kappa+1}}\left(\sqrt{J_{1}-J_{2}}-\sqrt{J_{1}+J_{2}}\right)
$$

\section{Results and discussion}

To evaluate the efficiency of the developed program, crack propagation simulation was done and the stress intensity factor for various geometries was evaluated and compared with other related studies.

\subsection{Single edge cracked plate under plane stress condition}

This geometry is a rectangular plate $(200 \mathrm{~mm} \times 100 \mathrm{~mm} \times 1 \mathrm{~mm})$ as shown in Fig. 2 with an initial crack $(a=30 \mathrm{~mm})$ is considered for 2-dimensional finite element analysis. The material properties of the aluminum alloy used in this study were taken as $E=72 \mathrm{GPa}, v=0.3$ and $K_{I c}=1297 \mathrm{~N} / \mathrm{mm}^{1.5}$, where $E$, $\gamma$ and $K_{I c}$ represents Young's modulus, Poisson's ratio and fracture toughness, respectively. The cracked plate is subjected under a uniform tensile load stress at both ends.

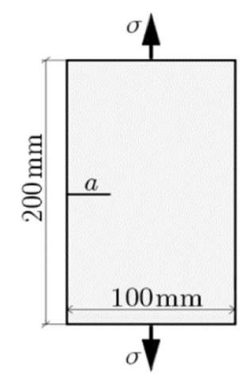

Fig. 2. Single edge cracked plate 
The initial mesh and the final crack growth direction are shown in Fig. 3a, 3b.

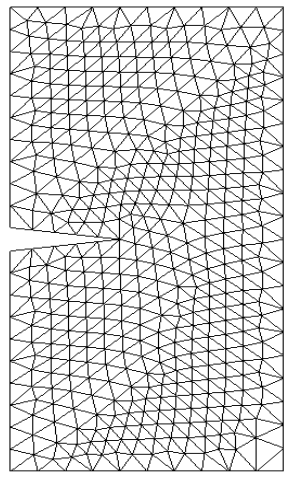

(a)

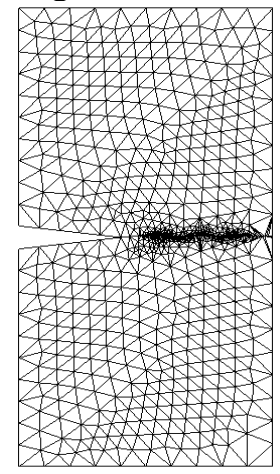

(b)

Fig. 3. (a): The final mesh; (b): crack path

This mesh will serve to calculate the stress intensity factors $K_{I}$ and $K_{I I}$ using the J-integral method based on Eq. (7).

The analytical stress intensity factor $K_{I}$ for this geometry is given by (Tada et al., 2000) as:

$$
K_{I}=F \sigma \sqrt{\pi \sigma}
$$

where $\mathrm{F}$ is the correction factor given by

$$
F=1.12-0.231\left(\frac{a}{w}\right)+10.55\left(\frac{a}{w}\right)^{2}-21.72\left(\frac{a}{w}\right)^{3}+30.39\left(\frac{a}{w}\right)^{4}
$$

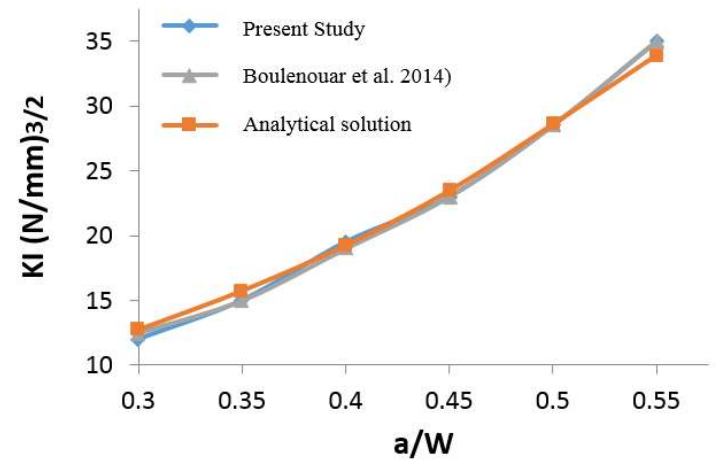

Fig. 4. Relationship between SIF $K_{I}\left(\mathrm{~N} / \mathrm{mm}^{3 / 2}\right)$ and the initial crack length size $(a / W)$

The calculated values of stress intensity factor has been compared to the analytical solution described in Eq. (9) as well as the numerical solution using finite element method obtained by (Boulenouar et al. 2014) as shown in Fig. 4 with excellent agreement. Fig. 5 shows the comparison with a good agreement between the present study results and other related results obtained by (Alshoaibi \& Ariffin, 2006) using FRANC2D/L program and finite element results using ANSYS software by (Boulenouar et al., 2014).

These results allow us to conclude that the numerical model used correctly describes the stress and deformation field near the crack tip, under pure mode I conditions. Fig. 6a and $6 \mathrm{~b}$ show the stress distribution in $\mathrm{Y}$ and $\mathrm{X}$ direction with enlargement at crack tip for the final step of the crack. 


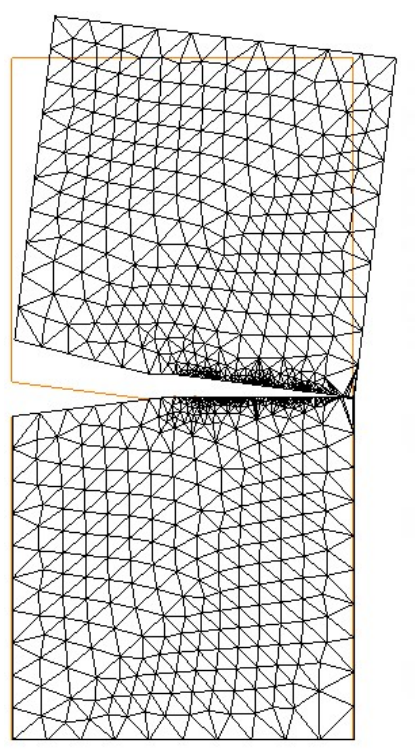

(a)

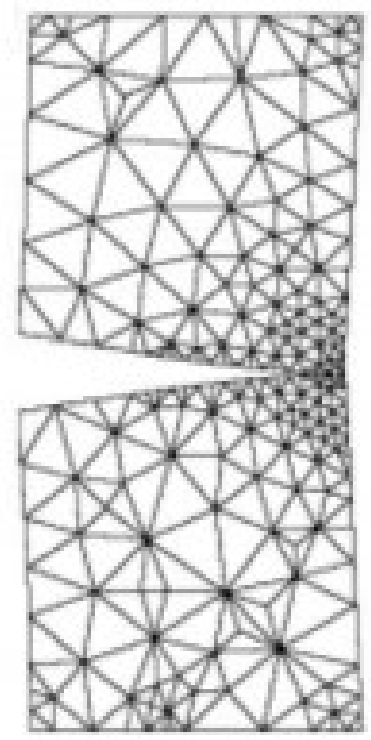

(b)

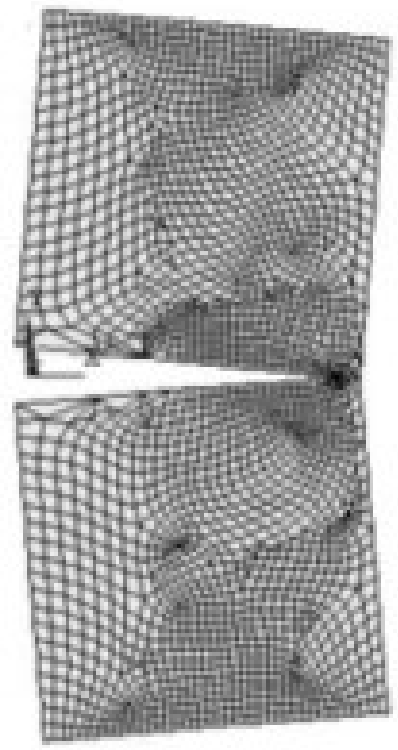

(c)

Fig. 5. Crack propagation path under mode I loading: (a) present study (b) (Alshoaibi and Ariffin 2006), (c) (Boulenouar et al., 2014)

\subsection{Cracked Plate with One Hole}

A rectangular plate with one hole of diameter $10 \mathrm{~mm}$ and one $6 \mathrm{~mm}$ long edge crack, as shown in Fig. 7a. The initial mesh for this geometry is shown in Fig. 7b. A tensile load of $10 \mathrm{MPa}$ is applied on the top of plate, and the plate is fixed at the bottom. The Young's modulus is $72 \mathrm{GPa}$, Poisson's ratio is 0.33 .

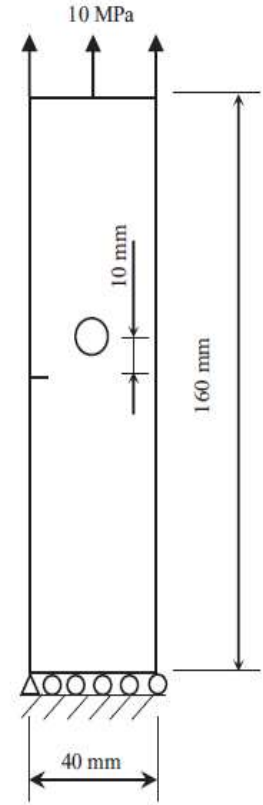

(a)

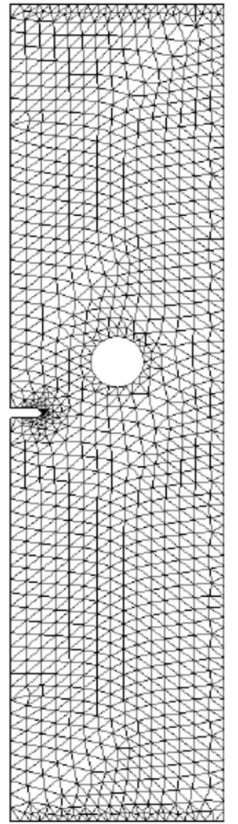

(b)

Fig. 7. (a) cracked plate with one hole and (b) Initial mesh 
The comparison of the result of the propagation path of the crack using the present study program with the experimental results obtained by (Varfolomeev et al., 2014) and BEM results reported by (Liu et al., 2017) are shown in Figs. 8a, 8b, and 8c. Very good agreement among the present study, experimental and BEM results are observed. As well as the contour of maximum stress is presented in Fig. 9.

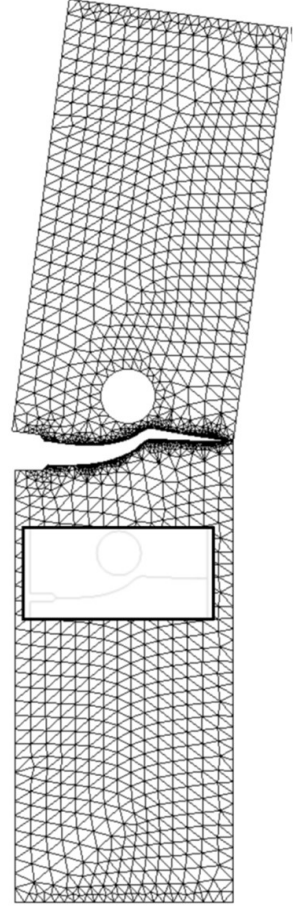

(a)

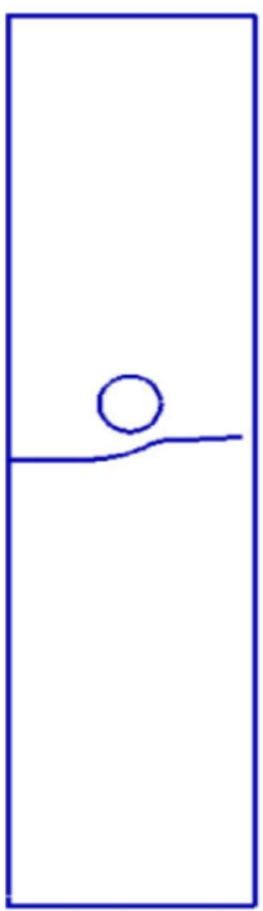

(b)

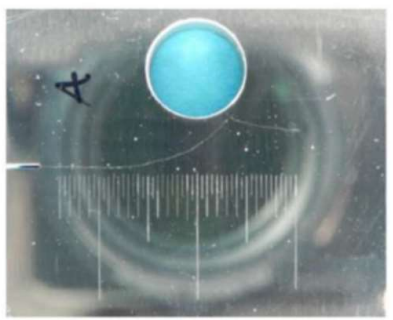

(c)

Fig. 8. (a) Present study, (b) BEM by (Liu et al. 2017) (c) Experimental work by (Varfolomeev et al., 2014)
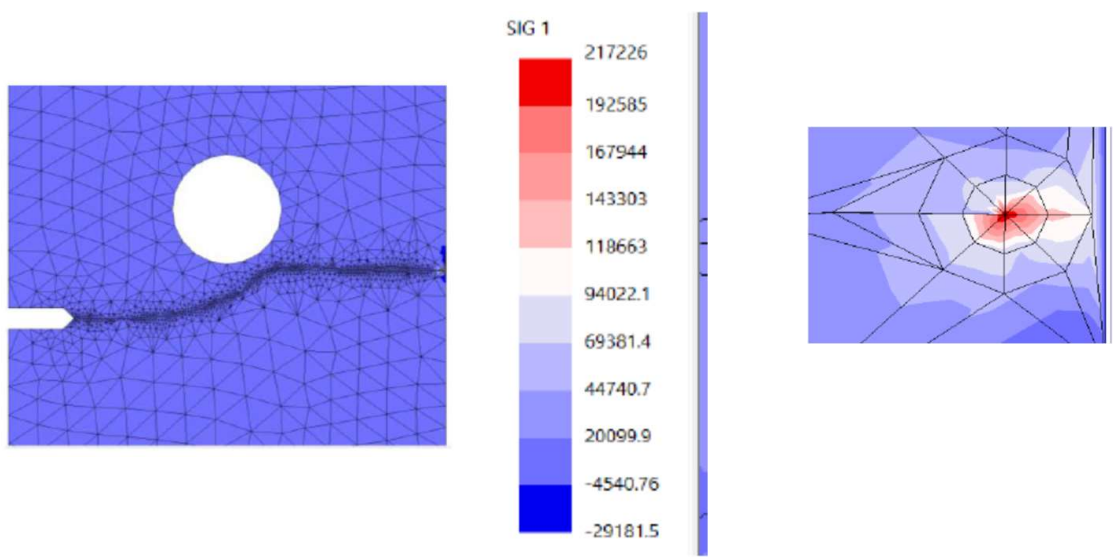

Fig. 9. Contour of $\sigma_{X}$ for cracked plate with one hole

\subsection{Tensile plate with central horizontal crack}

A test of a plate in plane strain state subjected to a tensile loading with a central horizontal crack is considered. The plate is a square of edge length $\mathrm{W}=10 \mathrm{~cm}$. The geometry can be inferred from Fig. 10 
for $\alpha=0^{\circ}$. The plate is loaded by a tensile distributed load of $\mathrm{P}=1 \mathrm{MPa}$, and is made of isotropic material with Young's modulus $\mathrm{E}=100 \mathrm{GPa}$ and Poisson coefficient $v=0.3$. Based on geometry and loading condition, the analytical equation of stress intensity factor is given by (Tada et al., 2000) as:

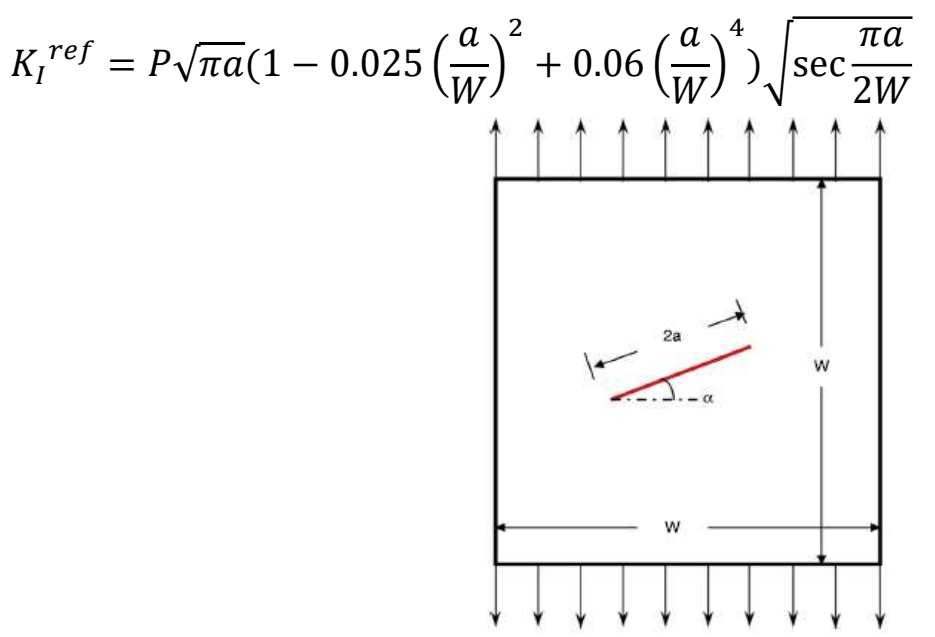

Fig. 10. Central inclined crack in a square plate of edge length $10 \mathrm{~mm}$ with crack length $2 \mathrm{a}$.

The present results for the normalized stress intensity factor with various initial crack lengths are shown in Fig. 11 which exhibit close agreement to the analytical solutions calculated by Eq. (11). Fig. 12 shows the crack propagation for $a / W=0.5$ in which the crack propagates towards the expected path under mode I loading condition.

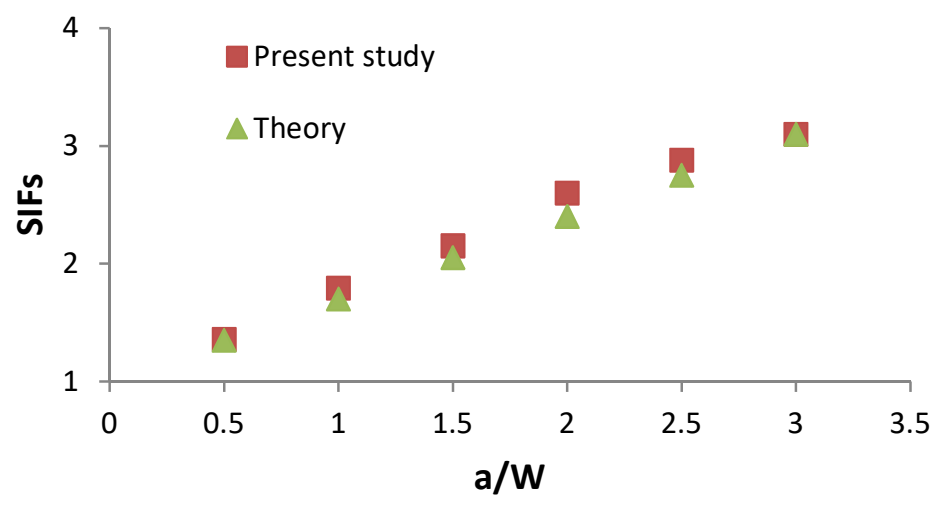

Fig. 11. Comparison between analytical solution of (Tada et al. 2000) and present study for the calculated SIFs with different value of $(\mathrm{a} / \mathrm{W})$ ratio

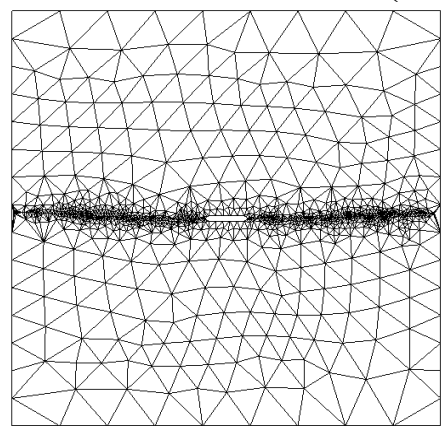

Fig. 12. The crack propagation trajectory under mode I loading 


\subsection{Slanted central crack}

The same geometry of last example section 4.3 but with the central crack of length 2 a inclined with angle $\alpha$ is shown in Fig. 10. For an infinite plate subjected to a plane strain state, the analytical solution predicts, the following values of the stress intensity factors obtained by (Benvenuti, 2017) as:

$$
K_{I}^{\text {ref }}=P \sqrt{\pi a} \cos ^{2} \alpha, \quad K_{I I}^{\text {ref }}=P \sqrt{\pi a} \sin \alpha \cos \alpha
$$

Table 1 shows the comparison between the results of stress intensity factors of present study to those from the analytical solutions of Eqs. (12).

Table 1. Dimensionless stress intensity factor for slanted central crack.

\begin{tabular}{lllll}
\hline$\alpha$ & Present study for $\boldsymbol{K}_{\boldsymbol{I}}$ & Present study for $\boldsymbol{K}_{\boldsymbol{I I}}$ & Analytical solution for $\boldsymbol{K}_{\boldsymbol{I}}$ & Analytical solution for $\boldsymbol{K}_{\boldsymbol{I I}}$ \\
\hline 0 & 1.358285 & 0.058559 & 1.252996409 & 0 \\
20 & 1.105888 & 0.40761 & 1.106566169 & 0.402535401 \\
40 & 0.735175 & 0.617298 & 0.735725176 & 0.616903128 \\
60 & 0.3137386 & 0.547073 & 0.313825356 & 0.542895653 \\
90 & -0.024982 & 0.000276 & $7.9457 \mathrm{E}-07$ & 0.000997794 \\
\hline
\end{tabular}

Fig. 13 shows the values of $K_{I}$ and $K_{I I}$ computed for variable inclination $\alpha$ and crack lengths $2 \mathrm{a}=$ $\mathrm{W} / 10$. The green and blue squares denote for present values for $K_{I}, K_{I I}$, the continuous blue and red lines denote the reference values Eq. (12), where for $\alpha=0$ the reference formula Eq. (12) has been exploited, being more accurate, and the blue and orange circles denote for (Benvenuti, 2017) results.

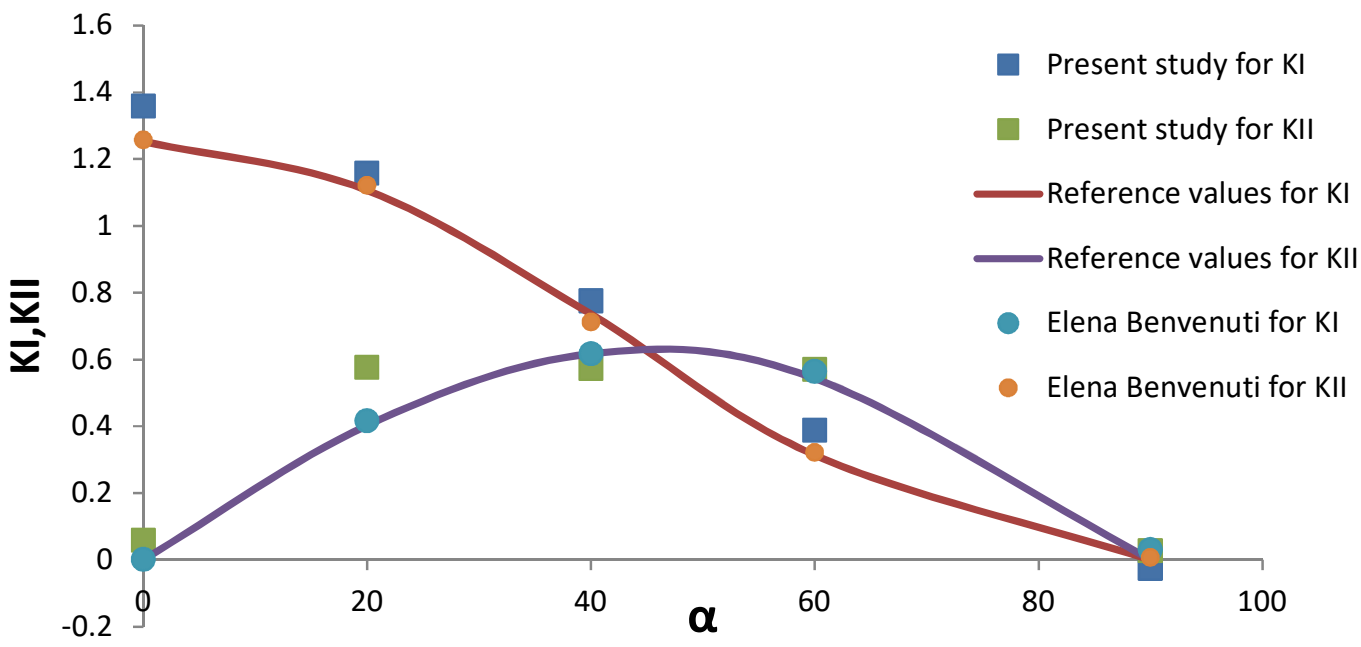

Fig. 13. Comparison of $K_{I}$ and $K_{I I}$ of CCT for variable inclination $\alpha$ and crack lengths $2 \mathrm{a}=$ $\mathrm{W} / 10$ between present work, reference values, and Benvenuti (2017) results.

\section{Conclusions}

The finite element method is appropriate to model problems involving fracture due to crack propagation. Therefore, two-dimensional finite element method on linear elastic fracture mechanics concepts were used in this work. Crack growth rates and stress intensity factors distributions along the crack fronts play an important parameter in the crack propagation analysis. Based on that the historical value of the stress intensity factors for both modes has been evaluated and compared successfully to other numerical results with a good agreement. The numerical simulations demonstrate the capability of the developed program to perform crack growth simulation successfully. 


\section{References}

Abd-Elhady, A. (2013). Mixed mode I/II stress intensity factors through the thickness of disc type specimens. Engineering Solid Mechanics, 1(4), 119-128.

Akbardoost, J., Ayatollahi, M. R., Aliha, M. R. M., Pavier, M. J., \& Smith, D. J. (2014). Size-dependent fracture behavior of Guiting limestone under mixed mode loading. International Journal of Rock Mechanics and Mining Sciences, 71, 369-380.

Aliha, M. R. M., \& Ayatollahi, M. R. (2008). On mixed-mode I/II crack growth in dental resin materials. Scripta Materialia, 59(2), 258-261.

Aliha, M. R. M., \& Ayatollahi, M. R. (2012). Analysis of fracture initiation angle in some cracked ceramics using the generalized maximum tangential stress criterion. International Journal of Solids and Structures, 49(13), 1877-1883.

Aliha, M. R. M., \& Rezaei, M. (2011). Experimental and theoretical study of fracture paths in brittle cracked materials subjected to pure mode II loading. In Applied Mechanics and Materials (Vol. 70, pp. 159-164). Trans Tech Publications.

Aliha, M. R. M., \& Saghafi, H. (2013). The effects of thickness and Poisson's ratio on 3D mixed-mode fracture. Engineering Fracture Mechanics, 98, 15-28.

Aliha, M. R. M., Ayatollahi, M. R., Smith, D. J., \& Pavier, M. J. (2010). Geometry and size effects on fracture trajectory in a limestone rock under mixed mode loading. Engineering Fracture Mechanics, 77(11), 2200-2212.

Aliha, M. R. M., Bahmani, A., \& Akhondi, S. (2016a). Mixed mode fracture toughness testing of PMMA with different three-point bend type specimens. European Journal of Mechanics-A/Solids, 58, 148162.

Aliha, M. R. M., Berto, F., Bahmani, A., \& Gallo, P. (2017). Mixed mode I/II fracture investigation of Perspex based on the averaged strain energy density criterion. Physical Mesomechanics, 20(2), 149156.

Aliha, M. R. M., Berto, F., Bahmani, A., Akhondi, S., \& Barnoush, A. (2016b). Fracture assessment of polymethyl methacrylate using sharp notched disc bend specimens under mixed mode I+ III loading. Physical Mesomechanics, 19(4), 355-364.

Alshoaibi, A. M., \& Ariffin, A. K. (2006). Finite element simulation of stress intensity factors in elasticplastic crack growth. Journal of Zhejiang University-SCIENCE A, 7(8), 1336-1342.

Atluri, S. N. (1982). Path-independent integrals in finite elasticity and inelasticity, with body forces, inertia, and arbitrary crack-face conditions. Engineering Fracture Mechanics, 16(3), 341-364.

Ayatollahi, M. R., \& Aliha, M. R. M. (2007). Wide range data for crack tip parameters in two disc-type specimens under mixed mode loading. Computational materials science, 38(4), 660-670.

Ayatollahi, M. R., \& Aliha, M. R. M. (2009). Analysis of a new specimen for mixed mode fracture tests on brittle materials. Engineering Fracture Mechanics, 76(11), 1563-1573.

Ayatollahi, M. R., Aliha, M. R. M., \& Hassani, M. M. (2006). Mixed mode brittle fracture in PMMAan experimental study using SCB specimens. Materials Science and Engineering: A, 417(1-2), 348356.

Ayatollahi, M. R., Aliha, M. R. M., \& Saghafi, H. (2011). An improved semi-circular bend specimen for investigating mixed mode brittle fracture. Engineering Fracture Mechanics, 78(1), 110-123.

Azócar, D., Elgueta, M., \& Rivara, M. C. (2010). Automatic LEFM crack propagation method based on local Lepp-Delaunay mesh refinement. Advances in Engineering Software, 41(2), 111-119.

Belytschko, T., \& Black, T. (1999). Elastic crack growth in finite elements with minimal remeshing. International journal for numerical methods in engineering, 45(5), 601-620.

Belytschko, T., Lu, Y. Y., \& Gu, L. (1994). Element-free Galerkin methods. International journal for numerical methods in engineering, 37(2), 229-256.

Benvenuti, E. (2017). An effective XFEM with equivalent eigenstrain for stress intensity factors of homogeneous plates. Computer Methods in Applied Mechanics and Engineering, 321, 427-454. 
Boulenouar, A., Benseddiq, N., Mazari, M., \& Benamara, N. (2014). FE model for linear-elastic mixed mode loading: estimation of SIFs and crack propagation. Journal of Theoretical and Applied Mechanics, 52.

Cooke, M. L., \& Pollard, D. D. (1996). Fracture propagation paths under mixed mode loading within rectangular blocks of polymethyl methacrylate. Journal of Geophysical Research: Solid Earth, 101(B2), 3387-3400.

Dehghany, M., Saeidi Googarchin, H., \& Aliha, M. R. M. (2017). The role of first non-singular stress terms in mixed mode brittle fracture of V-notched components: an experimental study. Fatigue \& Fracture of Engineering Materials \& Structures, 40(4), 623-641.

Duarte, C. A., \& Oden, J. T. (1996). H-p clouds - an h-p meshless method. Numerical Methods for Partial Differential Equations: An International Journal, 12(6), 673-705.

Guo, L., Xiang, J., Latham, J. P., \& Izzuddin, B. (2016). A numerical investigation of mesh sensitivity for a new three-dimensional fracture model within the combined finite-discrete element method. Engineering Fracture Mechanics, 151, 70-91.

Khan, S. M., \& Khraisheh, M. K. (2000). Analysis of mixed mode crack initiation angles under various loading conditions. Engineering Fracture Mechanics, 67(5), 397-419.

Khan, S. M., \& Khraisheh, M. K. (2004). A new criterion for mixed mode fracture initiation based on the crack tip plastic core region. International Journal of Plasticity, 20(1), 55-84.

Lan, M., Waisman, H., \& Harari, I. (2013). A High-order extended finite element method for extraction of mixed-mode strain energy release rates in arbitrary crack settings based on Irwin's integral. International Journal for Numerical Methods in Engineering, 96(12), 787-812.

Liu, Y. J., Li, Y. X., \& Xie, W. (2017). Modeling of multiple crack propagation in 2-D elastic solids by the fast multipole boundary element method. Engineering Fracture Mechanics, 172, 1-16.

Matvienko, Y. G. (2012). Maximum average tangential stress criterion for prediction of the crack path. International Journal of Fracture, 176(1), 113-118.

Miller, E. E., Sutton, M. A., Deng, X., Watts, H., Reynolds, A. P., Ke, X., \& Schreier, H. W. (2015). Experimental and predicted crack paths for Al-2024-T351 under mixed-mode I/II fatigue. In Fracture, Fatigue, Failure, and Damage Evolution, Volume 5 (pp. 11-20). Springer, Cham.

Mirsayar, M. M., Aliha, M. R. M., \& Samaei, A. T. (2014). On fracture initiation angle near bi-material notches-Effects of first non-singular stress term. Engineering fracture mechanics, 119, 124-131.

Mirsayar, M. M., Razmi, A., Aliha, M. R. M., \& Berto, F. (2018). EMTSN criterion for evaluating mixed mode I/II crack propagation in rock materials. Engineering Fracture Mechanics, 190, 186-197.

Rao, B. N., \& Rahman, S. (2001). A coupled meshless-finite element method for fracture analysis of cracks. International Journal of Pressure Vessels and Piping, 78(9), 647-657.

Razavi, S. M. J., Aliha, M. R. M., \& Berto, F. (2018). Application of an average strain energy density criterion to obtain the mixed mode fracture load of granite rock tested with the cracked asymmetric four-point bend specimens. Theoretical and Applied Fracture Mechanics, 97, 419-425.

Rice, J. R. (1968). A path independent integral and the approximate analysis of strain concentration by notches and cracks. Journal of applied mechanics, 35(2), 379-386.

Sabbagh-Yazdi, S. R., Farhoud, A., \& Gharebaghi, S. A. (2018). Simulation of 2D linear crack growth under constant load using GFVM and two-point displacement extrapolation method. Applied Mathematical Modelling, 61, 650-667.

Sutton, M. A., Boone, M. L., Ma, F., \& Helm, J. D. (2000). A combined modeling-experimental study of the crack opening displacement fracture criterion for characterization of stable crack growth under mixed mode I/II loading in thin sheet materials. Engineering Fracture Mechanics, 66(2), 171-185.

Tada, H., Paris, P., \& Irwin, G. (2000). The analysis of cracks handbook. New York: ASME Press, $2,1$.

Varfolomeev, I., Burdack, M., Moroz, S., Siegele, D., \& Kadau, K. (2014). Fatigue crack growth rates and paths in two planar specimens under mixed mode loading. International Journal of Fatigue, 58, 12-19.

Williams, M. L. (1957). The bending stress distribution at the base of a stationary crack. Trans. ASME, 79, 109-114. 
Xie, D., \& Waas, A. M. (2006). Discrete cohesive zone model for mixed-mode fracture using finite element analysis. Engineering fracture mechanics, 73(13), 1783-1796.

Zhang, R., \& Guo, R. (2018). Determination of crack tip stress intensity factors by singular Voronoi cell finite element model. Engineering Fracture Mechanics, 197, 206-216.

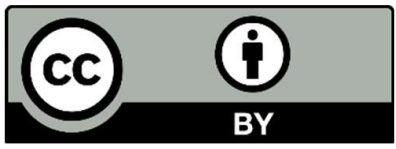

(C) 2020 by the authors; licensee Growing Science, Canada. This is an open access article distributed under the terms and conditions of the Creative Commons Attribution (CC-BY) license (http://creativecommons.org/licenses/by/4.0/). 